Nouvelles perspectives en sciences sociales

Avant-propos

Pistes d'exploration épistémologique de la réflexivité et de la distanciation critique

\title{
Denis Martouzet
}

Volume 13, numéro 1, novembre 2017

URI : https://id.erudit.org/iderudit/1044009ar

DOI : https://doi.org/10.7202/1044009ar

Aller au sommaire du numéro

Éditeur(s)

Prise de parole

ISSN

1712-8307 (imprimé)

1918-7475 (numérique)

Découvrir la revue

Citer ce document

Martouzet, D. (2017). Avant-propos : pistes d'exploration épistémologique de la réflexivité et de la distanciation critique. Nouvelles perspectives en sciences sociales, 13(1), 15-19. https://doi.org/10.7202/1044009ar d'utilisation que vous pouvez consulter en ligne.

https://apropos.erudit.org/fr/usagers/politique-dutilisation/ 


\title{
Avant-propos
}

\section{Pistes d'exploration épistémologique de la réflexivité et de la distanciation critique}

\author{
Denis Martouzet \\ Université François-Rabelais, Tours
}

es textes composant ce numéro entrent tous dans les thémaLtiques de la réflexivité et de la distanciation critique : la recherche est elle-même questionnée comme objet de recherche. D'emblée, cependant, nous pouvons constater une très grande hétérogénéité des articles proposés, hétérogénéité relative aux différentes manières d'appréhender cet objet de recherche qu'est la recherche. Ces dix textes peuvent donc être lus de façon totalement autonome les uns par rapport aux autres. Chacun propose une brique et les auteurs ont respecté leur "part de contrat ", individuellement ou par petits groupes, lorsqu'il y a des coauteurs, et nous les en remercions. Les conditions de leur travail d'écriture et notamment l'isolement de chacun ont conduit au caractère de complétude de chaque article.

Le lecteur saura saisir cela mais immédiatement il percevra combien l'ensemble de ces articles, alors qu'ils sont, chacun, complets et autonomes, montre que le champ de la recherche comme objet de recherche n'a été ici qu'effleuré et se révèle très vaste. Prenons une métaphore géographique. Chaque texte peut 
être considéré à l'image d'un village, petit lieu qui met en relation des éléments locaux et qui s'inscrit dans un environnement proche qui l'alimente. Chaque auteur est villageois de son village. Le lecteur, s'il va au bout de sa lecture, saura qu'il y a dix villages. Plus encore, il saura que certains de ces villages s'alimentent de la même façon, empruntent aux mêmes environnements. Il poussera la réflexion jusqu'à mettre en évidence des pistes permettant d'aller, plus ou moins facilement, d'un village à l'autre, de suivre, d'esquisser, de créer des liens entre ces textes, liens deux à deux ou, parfois, plus largement. La connaissance de ces chemins l'amènera à considérer l'ampleur du travail d'exploration qu'il s'agit encore de faire pour avoir une connaissance relativement complète de la forêt qui enserre ces villages.

Nous proposons donc au lecteur d'avoir une lecture de mise en perspective des textes les uns par rapport aux autres et nous l'y invitons en ouvrant quelques pistes (de réflexion) permettant de les relier.

La première est celle du rapport à soi qu'entretient le chercheur. La question du chercheur est alors de savoir qui est le chercheur - plutôt d'essayer d'en cerner la silhouette -, arrivant rapidement à la conclusion qu'il y a plusieurs personnes en la personne du chercheur. Cela est particulièrement vrai pour les doctorants qui ont réalisé leur thèse dans le cadre d'une bourse CIFRE. Au-delà du statut ambigu du doctorant-chercheur-salarié auquel conduit ce dispositif de financement des travaux doctoraux, il s'agit dans un premier temps de s'assurer du rôle qu'il convient de prendre pour mener à bien "sa " recherche et, rapidement, dans un second temps, de se positionner - en tant que personne, salarié (donc collègue, inférieur hiérarchique...), chercheur, mais chercheur apprenti puisque doctorant - pour déchiffrer comment toutes ces personnes, en une seule, cohabitent et s'influencent de diverses façons. Il y a ainsi plusieurs soi en soi. La présence accentuée du « je » dans ces articles en est l'indice, les formes plus neutres généralement utilisées n’ont pas la force évocatrice du "je " comme unité renvoyant à une diversité : derrière la personne et avec elle cohabitent le chercheur, 
l'enquêteur... Le texte de Marion Bourhis est de ce point de vue le plus explicite, mais l'on trouve de grandes similarités avec celui de Camille Rouchi, autre chercheur.e ayant réalisé son doctorat dans le cadre d'une telle convention. Toutes deux en tirent l'idée que ce qui est ou apparaît comme contrainte ou difficulté de gestion de la relation entre l'objet de recherche et l'environnement de recherche devient, par l'explicitation de ce système complexe, source de savoir. Ce rapport à soi peut même être renforcé par les affects, comme le soulignent Souheil Essid et Yosra Essid ou, de façon plus volontaire, par l'engagement de soi - en tant que personne et non en tant que chercheur - dans la recherche elle-même. Le texte de Cyril Blondel évoque cela. Celui de Marion Bourhis aussi et l'on pourrait généraliser le propos à toute recherche qui est recherche-action, à l'image du texte de Julie Descheneaux et al. ou aux recherches qui se donnent aussi, explicitement ou non pour le lecteur, le statut de plaidoyer. Ce sont là le texte de Lionel Francou et Antoine Delporte et, de façon moins visible, celui de Dominique Loiseau.

La deuxième piste de réflexion permettant de relier ces textes est le rapport à l'autre. Le rapport à soi n'a d'effectivité que s'il s'inscrit dans une relation avec le rapport à l'autre. Le pur narcissisme n'existe pas, en recherche comme dans tout autre cadre social de production. Moins évidemment présent que le rapport à soi, le rapport à l'autre s'exprime notamment, dans les textes qui suivent, en termes d'empathie, particulièrement dans la relation entre le chercheur ou l'enquêteur et la personne enquêtée. Élargissant ce cadre assez classique, Lionel Francou et Antoine Delporte vont jusqu'à proposer une sociologie qui se devrait d'être empathique, tandis que Dominique Loiseau intègre la notion dans celle, plus large, d'altérité, dépassant l'empathie pour aller jusqu'à la connivence, le non-chercheur obtenant alors le statut de sujet agissant dans la recherche, n'étant plus simple objet d'analyse, voire simple outil pour capter de l'information à analyser. La réflexivité et la distanciation critique montrent clairement le caractère flou des notions de soi, d'altérité, etc. et leur porosité. 
Ce rapport à l'autre s'inscrit plus largement dans le rapport aux différents cadres dans lesquels s'effectuent les recherches ici restituées et réanalysées. Tout d'abord, le cadre académique peut faire l'objet d'un examen critique et, si les règles méthodologiques permettent la clarification et la délimitation du champ de validité des résultats, elles conduisent aussi à une forme de normalisation qui rend plus difficile l'expérimentation, l'innovation, la relation à d'autres cadres institutionnels, professionnels ou culturels : Nathalie Brevet, chercheur.e et artiste, montre que le lien entre ces deux mondes ne peut être fait que de brèves rencontres, ce qui ne signifie pas qu'elles sont sans lendemain. Dominique Loiseau dénonce un entre-soi du monde académique qu'incite la nécessité d'une intégration professionnelle du chercheur (Camille Rouchi) permettant d'obtenir une reconnaissance par les pairs (Florence Bétrisey). Mais ce respect du cadre ou le simple conformisme, s'ils ont leur utilité, sont mis en balance avec des possibilités de circulation, d'allers et retours entre mondes généralement parallèles, d'innovation, d'inventivité, de bricolage méthodologique. La rigueur de ce monde académique est renforcée par le cadre culturel d'origine du chercheur, y compris à ses dépens. Plus encore, le cadre académique, par ses routines, ses normes et leur non-remise en question, rend invisible le cadre culturel. Cyril Blondel parle ainsi de " colonialité » du savoir alors que Florence Bétrisey cible son propos sur l'eurocentrisme du chercheur.

Ces différents rapports (à soi, à l'autre, aux mondes et autres cadres) influent sur la construction de l'objet de recherche, plus exactement, sur la représentation que le chercheur a de l'objet qu'il a construit. Cette construction amène le chercheur luimême à adopter une posture ou à se doter d'un appareillage critique vis-à-vis de lui-même pour saisir en quoi son objet de recherche est un construit subjectif social. En retour, le chercheur s'en trouve changé et la prise de conscience de ce changement rejaillit sur son objet et l'outillage qu'il met en œuvre. Tous les auteurs des textes de ce numéro thématique abordent cette question de l'outillage. Florence Bétrisey utilise une démarche 
auto-ethnographique qui met au centre de son propos le rapport à soi ; très proches, Souheil Essid et Yosra Essid montrent combien la démarche de l'observation ethnographique rejaillit sur le chercheur, allant jusqu'à évoquer une désaffiliation ; Isabelle Lefort et Laura Péaud insistent sur l'appareillage conceptuel et la manière dont celui-ci, par son évolution, a conduit la géographie, lorsqu'elle s'examine elle-même, à passer d'une approche historique à une approche épistémologique ; de son côté, Marion Bourhis, partant des multiples soi qui la composent, met en évidence la nécessité d'une approche par la complexité, conclusion à laquelle parviennent, par un autre chemin, celui du lien entre recherche et pratique, Julie Descheneaux et al. ; Nathalie Brevet parle d'expérimentation, ce qui renvoie nécessairement à des outillages particuliers, ce que Dominique Loiseau envisage comme moyen de sortir de l'entre-soi académique.

Ces quelques pistes à peine esquissées ici permettent d'explorer le continent de l'épistémologie en action, d'en saisir intuitivement l'ampleur. Le lecteur saura sans nul doute trouver ses propres chemins de traverse. 